\title{
The Effects of Calcium Excess, Water Amount and Mixing Time on The Injectability of Calcium Phosphate Filling Materials
}

\author{
A.S.F. Alqap ${ }^{1,2, a}$, I. Sopyan ${ }^{1, b}$, M. Husni ${ }^{1, c}$ and N. Athirah ${ }^{1, d}$ \\ ${ }^{1}$ Department of Manufacturing and Materials Engineering, International Islamic University Malaysia, \\ PO Box 10 Kuala Lumpur, Malaysia \\ ${ }^{2}$ Mechanical Engineering Program, University of Bengkulu, 38000 Bengkulu, Indonesia \\ aasepsofwan4@gmail.com, bsopyan@iium.edu.my, 'cfilamni_wassalam@yahoo.com, \\ dathirah918@yahoo.com
}

Keywords: Calcium Phosphate, Paste, Hydrothermal, Injectability, Resorbable, Calcium Excess, Water Amount, Mixing Time.

\begin{abstract}
Variation of calcium excess, water content and mixing time have been taken into the parameters of the injection test of calcium phosphate paste synthesized via low temperature hydrothermal method. The result indicated that all the parameters are very prominent to influence the material injectability possible to be fully injected out the syringe. Strict control of paste synthesizing parameters has successfully overcome poor injectability of the material.
\end{abstract}

\section{Introduction}

Calcium phosphate (CP) biomaterial is clinically accepted for implant fixation, augmentation, and bone substitution purposes. Many types of $\mathrm{CP}$ biomaterials have been used for biomedical application, as coating, particulates, blocks, porous and paste [1-4]. For bone filling material applications, calcium phosphate cement is more attractive than hydroxyapatite ceramic granules or powders because they can be moulded and shaped to fill intricate bony cavities or narrow dental defect sites. CP cement is mostly introduced in paste form and to deliver the material requires needles/applicators to access bony defect. The available formula of commercial CP cement made of tetracalcium phosphate (TTCP) and $\alpha$-tricalcium phosphate $(\alpha-\mathrm{TCP})$ always transform to hydroxyapatite (HA) when it is in contact with water, henceforth apatite cements.

Setting time that CP cement needs to harden is always concerns of many researches. Many factors affect setting time of CP cement, i.e. CP precursors, particle size, reagent solution [5-7]. The pressure required to inject $\mathrm{CP}$ cement is sometimes too high, and the clinician is therefore not able to force the cement through the cannula [8]. If the cement is injected while it is too liquid, the cement spread is not predictable and the risk of leakage increases. On the other hand, if the cement is too viscous, the pressure required for injection may be too high [8-10]. In most cases, apatite cements form a paste within five minutes, start to harden in situ within about $10 \mathrm{~min}$ and an optimal hardness is obtained after about 12 to $24 \mathrm{~h}$ [11]. And after setting, the apatite as the less resorbable materials remain dense and do not provide rapid bone substitution, hence, the use of resorbable cement materials is an advantage [11].

CP cement when sets initially may not be deformed unless with damaging its structure, furthermore, may not be scratched [12]. Meaning when it sets it can not longer be injected. And really, for example the 5:2 P/L cement with powder precursors of $\alpha-\mathrm{TCP}, \mathrm{Di}-\mathrm{CP}, \mathrm{HA}$ and CaCO3 is not injectable after 5 min mixing time where liquid is sodium phosphate acid [13]. However Vlad et al. demonstrated that calcium sulphate hemihydrate cement with $2 \mathrm{ml}$ solution per $1 \mathrm{~g}$ powder can be successfully injected even after 32 min mixing time [14].

By varying excesses of calcium precursor, as we reported elsewhere [15,16], CP materials have Ca-deficient apatite (CDA) phase after synthesis and it converts to different phases upon calcination, i.e. pure $\beta$-TCP, pure apatite or biphasic of apatite $-\beta$-TCP. CDA and $\beta$-TCP are known as resorbable 
phases. The present work aims at investigating injectability of $\mathrm{CP}$ cements produced by low temperature hydrothermal method as functions of calcium excess, water content and mixing time to find possible conditions for injecting these cements into bony cavity.

\section{Materials and Methods}

The $\mathrm{CP}$ biomaterial was prepared using calcium oxide granules $(\mathrm{CaO})$ (Techno Pharmchem, India) and ammonium $d i$-hydrogen phosphate (NH4H2PO4) (Systerm, Malaysia) as the precursors with distilled water as the solvent. For the preparation of the solution, a stoichiometric weight of calcium oxide was mixed with distilled water with vigorous stirring to get a soluble suspension. Into this suspension, ammonium di-hydrogen phosphate powder on the base of $1.67 \mathrm{Ca} / \mathrm{P}$ was then added dropwise. The synthesis temperature was between $80-100^{\circ} \mathrm{C}$ up to paste was obtained. Four synthesized pastes of different Ca-excess were prepared: 1,2, 10 and $20 \% \mathrm{CaO}$ excess. The paste was then treated into two groups: with drying and without drying. The first, it was dried overnight at $80^{\circ} \mathrm{C}$ in an oven. The dried powder was then crushed with a pestle in a mortar and sieved with a kitchen sieve before mixing with water for further extrusion test. And the second, another paste of the $20 \%$ $\mathrm{CaO}$ excess was kept as paste and when the weight was $80 \%$ of yield (with the range of $-0 \% /+10 \%$ ) then the synthesis process was stopped and the paste was brought for the extrusion test.

The injectability test was done using extrusion test following method outlined by Driessens et al. or Vlad et al. [13,14], by extruding non-needle $5 \mathrm{ml}$ polyethylene syringes filled with the mixture of dried powder and distilled water of 3:2 and 1:1 mass ratios for the first group or the as paste for the second. The extrusion was done using a Lloyd universal testing machine, LR $10 \mathrm{~K}$ model, at a crosshead speed of $50 \mathrm{~mm} / \mathrm{min}$ and a maximum load of $300 \mathrm{~N}$. The evolution of the extrusion force was recorded against the extruding time for different mixing or waiting times. For the first group, a predetermined weight of water as the above ratio was poured to predetermined weight of powder then they were being mixed using the pestle in the mortar with mixing times of 5 to $30 \mathrm{~min}$, after every 5 min interval the mixture was then injected. For the second group, i.e. the $20 \% \mathrm{CaO}$ excess paste, as aforementioned weight, was brought to extrusion after different waiting times. The mixing process of the first group was necessary to make water and powder a homogenous mixture while the second group was as a homogenous mixture after synthesis so no mixing anymore was necessary.

\section{Results and Discussion}

Fig. 1 shows the extrusion test of $\mathrm{CP}$ powder with different $\mathrm{Ca}$ excess when 1 part of water are mixed to 1 part of CP powder. Fig. 1a tells us four steps of loading. First, when loading is applied initially, ejection needs a near zero load when paste is compacted (compaction step). Secondly, after completely compacted, the ejection increases to a very low load while paste is being pushed out the syringe (extrusion step). When the volume of the paste is almost completely ejected out, loading force becomes increased because the ejector works onto a dead part (completion step). Finally the ejection automatically stops when the load comes to 300 N. Fig. 1a and Fig. $1 \mathrm{~b}$ show the ejection is success to completely eject paste out the syringe with very low extrusion loads. However, when $\mathrm{Ca}$ excess is more, the extrusion load is increased with mixing time increases (Fig. 1c and Fig, 1d). CP20 shows the fact that after $30 \mathrm{~min}$ no paste can be ejected out. The figure suggests that the water content here causes the material can be completely injected with very low loading even for high Ca excess when the mixing time is up to maximum $10 \mathrm{~min}$.

Fig. 2 shows the less water, i.e. 1 part of water, is given to 1.5 part of $\mathrm{CP}$ powder. $15 \mathrm{~min}$ is mixing time possible to inject $\mathrm{CP} 1$ through the syringe (Fig. 2a) but with higher load. CP2 as higher $\mathrm{Ca}$ excess is injectable after 10 min mixing with lower loading. For the higher Ca excess, injection loads are soon such increasing even after 5 min that the material is not longer injectable at 100 N. From Fig. 1 and Fig. 2, CP2 posses good injectability even better than CP1, the lower Ca excess. CP2 may have specific volume lower compared to the others. For both the ratios of water amounts, CP2 is always wetter while the others not that show by a fixed specific volume of water, the specific volume of CP2 
is lower compared to others. In other word, CP2 is higher in density than the others. By Archimedes method the density of CP1, CP2, CP10 and CP20 when molded by handy ejection into a $6 \mathrm{mmD} x$ $12 \mathrm{mmH}$ Teflon mould is $0.965,1.139,0.756$ and 0.714 respectively as calculated from dry weight divided by difference of dry weight and submerged weight in water multiplied with water density.

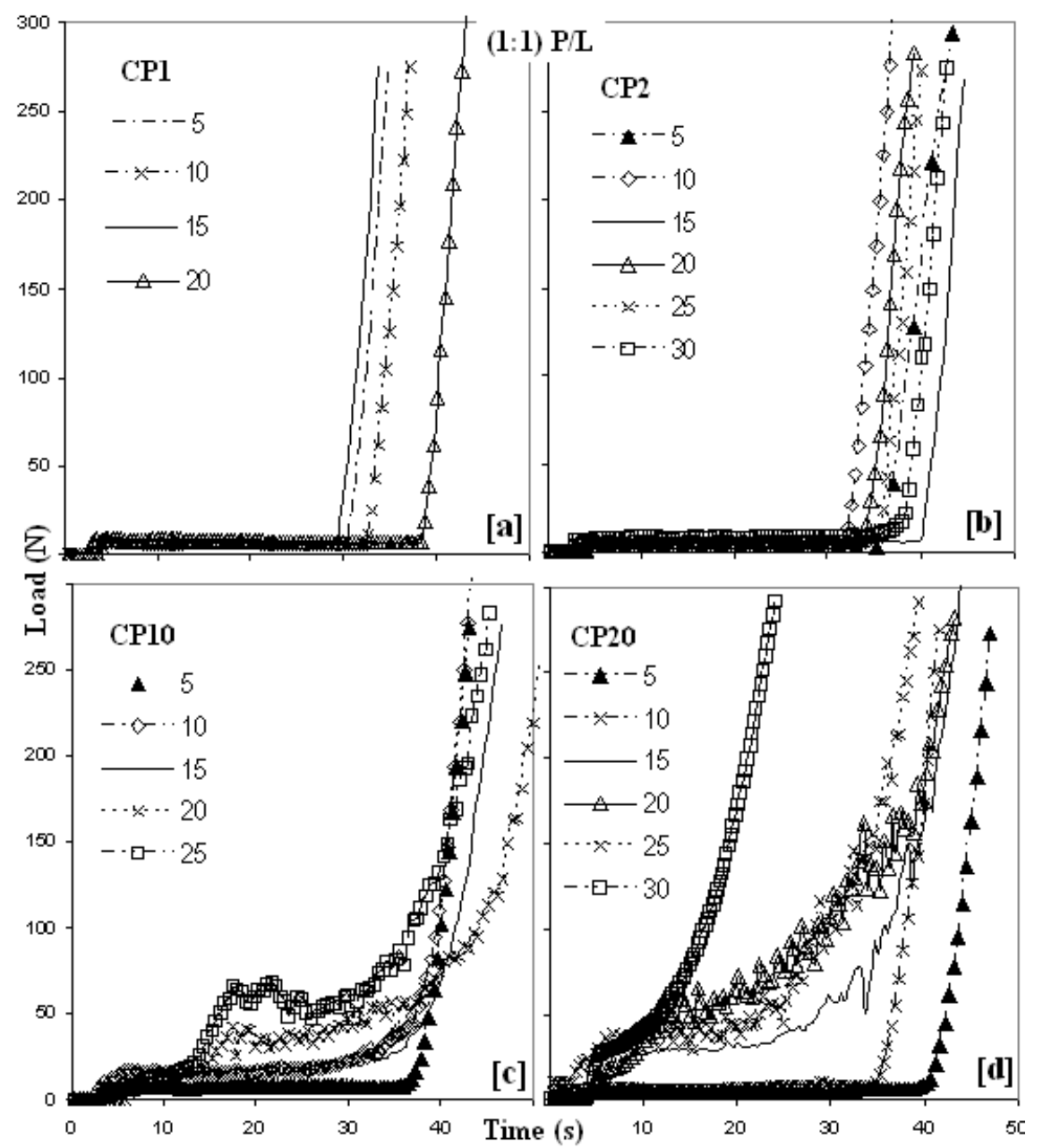

Fig. 1: Injection test on $\mathrm{CP}$ with different $\mathrm{Ca}$ excess with 1:1 $\mathrm{P} / \mathrm{L}$ ratio after different mixing times.

Fig. 2c and Fig. 2d show CP20 is not possible to injecting after 15 min mixing time with low load. The lower injectability of CP20 characterizes its rougher and non flexible structure as Fig. 3 (upper) shows. Fig. 3 shows the more in injectability, the more moldable, the finer in structure and the longer. Fig. 2c and Fig. 2d show the higher Ca excess is the lower injectability. However, Fig. 4 shows best finding, when CP20 is produced as Paste it is injectable with the injection load is lower than $50 \mathrm{~N}$ to push completely out the syringe, even after $60 \mathrm{~min}$ mixing time. This fact shows that the condition of Paste is promising method to prepare cement material such very flexible that surgeon is not worry from wasting the material.

The test has successfully shown the effect of calcium excess, water amount and mixing time on material possibility to push out the syringe without needle. However, handy-mixing and non uniform of powder particle may cause less reproducible of the test as compared to Vlad et al. work where an agitation machine for mixing was employed [14]. 


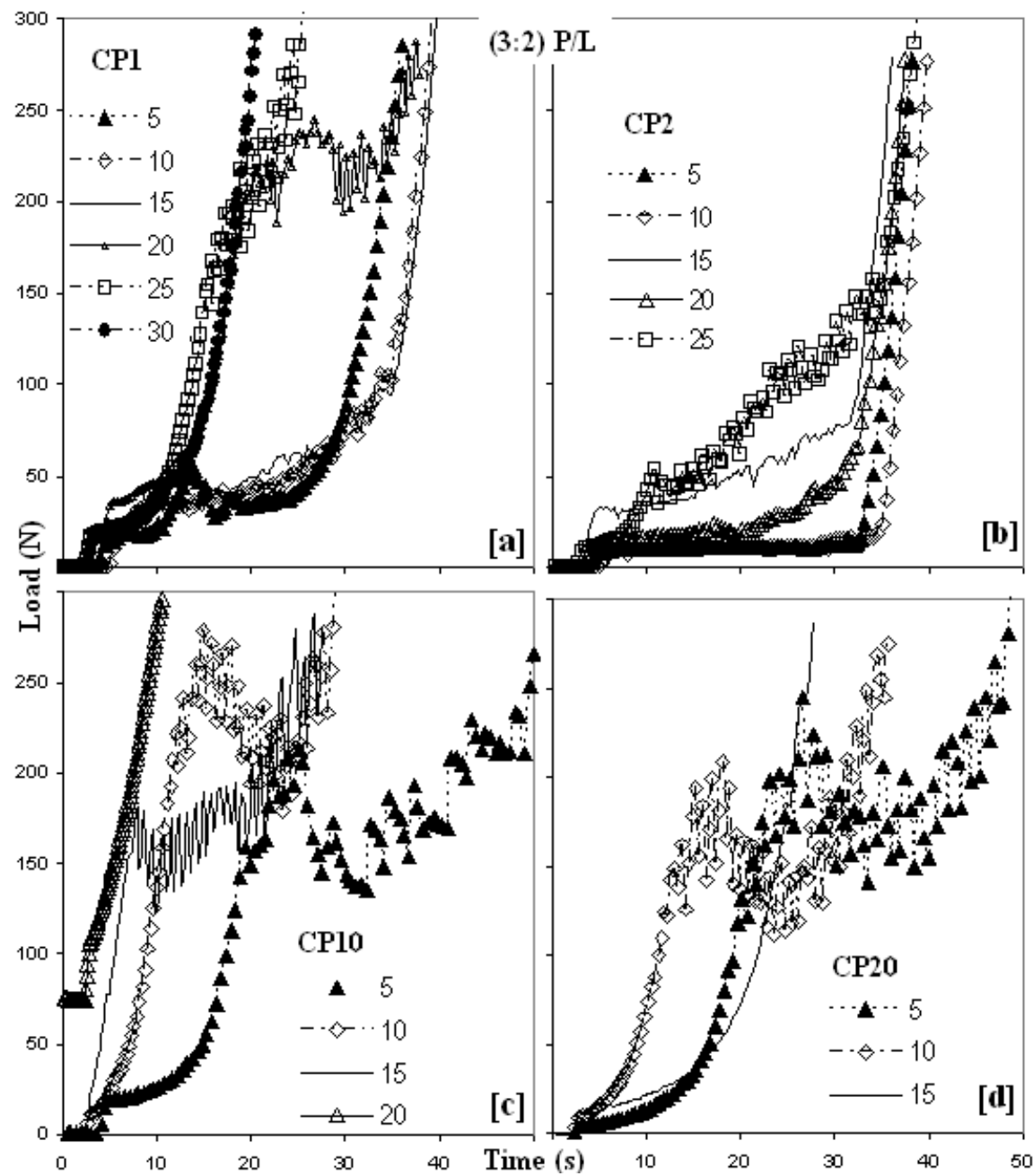

Fig. 2: Injection test on $\mathrm{CP}$ with different $\mathrm{Ca}$ excess with 3:2 $\mathrm{P} / \mathrm{L}$ ratio after different mixing times.

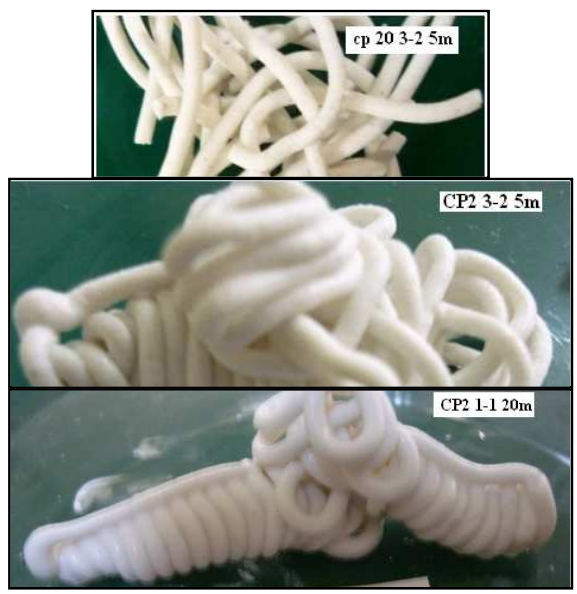

Fig. 3: Moldable form of Material after injection: CP20 (3:2) after 5 min [Upper]. CP2 (3:2) after 5 min [Middle]. CP2 (1:1) after $20 \mathrm{~min}$ [Lower].

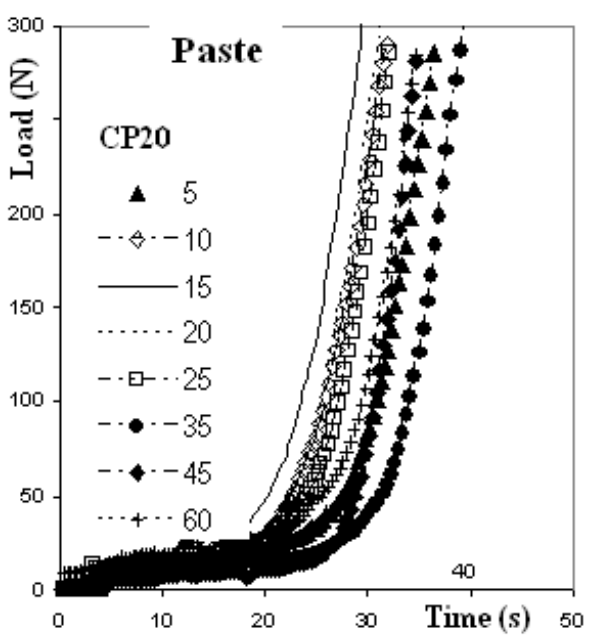

Fig. 4: CP20 Paste is fully injectable even after 60 min waiting time. 


\section{Summary}

The injection test by extruding $\mathrm{CP}$ biomaterial through the syringe without needle has been successfully done to elucidate the effects of $\mathrm{Ca}$ excess, water amount and mixing time. The results show that the increase in mixing time makes the sample delayed to eject completely out the syringe or the longer in mixing time the faster to increase the ejection loading. The more Ca excess the lesser the material can be completely injected. However, the Paste processing technique may overcome this limitation, if high $\mathrm{Ca}$ excess is necessary to design a phase such resorbable that causes bone remodeling takes place faster.

\section{Acknowledgment}

This work was supported by IIUM Research Grant No. EDW B 11-028-0506. One of the authors, (ASFA) thanks the Ministry of National Education, The Republic of Indonesia for the scholarship, No. 2865.33 \& 2866.38/D4.4/2008.

\section{References}

[1] I. Sopyan, M. Mel, S. Ramesh and K. A. Khalid: Sci. Technol. Adv. Mater. Vol. 8 (2007), p. 116

[2] S. Ramesh, C.Y. Tan, S.B. Bhaduri, W.D. Teng and I. Sopyan: J. Mater. Proces. Technol. Vol. 206 (2008), p. 221

[3] I. Sopyan and J. Kaur: Ceram. Int. Vol. 35 (2009), p. 3161

[4] J.A. Toque, M.K. Herliansyah, M. Hamdi, A. Ide-Ektessabi and I. Sopyan: J. Mech. Behavior Biomed. Mater. Vol. 3 (2010), p. 324

[5] F.C.M Driessens, M.G Boltong, E.A.P de Maeyer, R. Wenz, B Nies and J.A. Planell: Biomaterials Vol. 23 (2002), p. 4011

[6] M.P. Ginebra, F.C.M. Driessens and J.A. Planell: Biomaterials Vol 25 (2004), p. 3453

[7] M. Bohner: J. Mater. Chem. Vol. 17 (2007), p. 3980

[8] M. Bohner, B. Gasser, G. Baroud and P. Heini: Biomaterials Vol. 24 (2003), p. 2721

[9] G. Baroud, C. Matsushita, M. Samara, L. Beckman and T. Steffen: J. Biomed. Mater. Res. Appl. Biomater. Vol. 68B (2004), p. 105

[10] M. Komath and H.K. Varma: Bull. Mater. Sci. Vol. 26 (2003), p. 415

[11] G. Daculsi, R. LeGeros, M. Durand, P. Borget, S. Baroth, E. Goyenvalle, E. Aguado and F. Jegoux: J. Australian Ceram. Soc. Vol. 46 (2010), p. 1

[12] I. Khairoun, M.G. Boltong, F.C.M. Driessen and J.A. Planell: Biomaterials Vol. 18 (1997), p. 1535

[13] I. Khairoun, M.G. Boltong, F.C.M. Driessens and J.A. Planell: J. Mater. Sci. Mat. Med. Vol. 9 (1998), p. 425

[14] M. D. Vlad, R. Torres, J. Lopez, M. Barraco, J. A. Moreno and E. Fernandez: J. Mater. Sci. Mater. Med. Vol. 18 (2007), p. 347

[15] A.S.F. Alqap, I. Sopyan and S.A. Zubir: Adv. Mater. Res. Vol. 93-94 (2010), p. 405

[16] A.S.F. Alqap and I. Sopyan: Indian J. Chem. Vol. 48A (2009), p. 1492 
Mechanical and Aerospace Engineering

10.4028/www.scientific.net/AMM.110-116

The Effects of Calcium Excess, Water Amount and Mixing Time on the Injectability of Calcium Phosphate Filling Materials

10.4028/www.scientific.net/AMM.110-116.8 